\title{
The prognosis of trauma among children and the factors contributing to it
}

\author{
Hojjat Derakhshanfar ${ }^{1}$, Hamidreza Hatamabadi ${ }^{1,2^{*}}$, Kiandokht Karimian $^{1}$, Ali Abdalvand ${ }^{1}$, \\ Ali Arhami Dolatabadi ${ }^{1}$, Ali Shahrami ${ }^{1}$, Majid Shojaee ${ }^{1}$ \\ ${ }^{1}$ Emergency Medicine Department, Shahid Beheshti University of Medical Sciences, Tehran, Iran; \\ *Corresponding Author: hhatamabadi@yahoo.com \\ ${ }^{2}$ Safety Promotion and Injury Prevention Research Center, Shahid Beheshti University of Medical Sciences, Tehran, Iran
}

Received 2 February 2012; revised 24 February 2012; accepted 6 March 2012

\begin{abstract}
Background: The role of prognosis-prediction by scoring systems is an issue of importance to decrease the mortality rate in children with trauma. In this study, our goal was to evaluate the prognosis of trauma among children younger than 17 years of age and the contributing factors in the setting of a teaching hospital. Materials and Methods: In this descriptive cross-sectional study, we evaluated 151 consecutive children, younger than 17 years of age, victims of trauma who were admitted to a teaching hospital in Tehran, Iran between November 2009 and March 2010. Results: In this study, the mean PTS was $\mathbf{1 1 . 0 4}$ that this variable was 7.58 in cases with morbidity which was significantly higher than non-morbid cases $(P=0.001)$. Conclusions: The result of our study indicates that pediatric trauma scoring system can be used as a tool to predict the prognosis of trauma in children.
\end{abstract}

Keywords: Children; Trauma; Prognosis; Pediatric Trauma Score

\section{INTRODUCTION}

Injury is the main cause of mortality among children older than 1 year old [1]. Accidents account for $65 \%$ of mortal injuries in children younger than 19 years [2]. In the US, approximately 20,000 children and teenagers die annually as a result of injury [3]. And the real impact of injuries on the health of children will be better realized knowing that for every child who dies from an injury, 40 others are hospitalized and 1120 are admitted in emergency departments [3].

Age and sex are the most important factors affecting the patterns of injury and male children younger than 18 years have higher injury and mortality rates [4]. While falls are the most common injuries in the infants and tod- dlers, bicycle-related accidents are the main cause of injury in older children and adolescents [3].

Most cases of pediatric trauma occur as a result of blunt trauma, with penetrating injury accounting for $10 \%$ - $20 \%$ of all pediatric trauma admissions at most of the trauma centers $[1,2]$. The clinician should have a wellplanned and diligent approach and cast a net wide enough to catch all possible injuries [5]. In this era the role of prognosis-prediction by scoring systems is an issue of importance to decrease the mortality rate in traumatized children [6-8]. Accordingly, this study was done to determine the prognosis of traumatized children younger than 17 years of age and contributing factors in a teaching hospital with especial focus on pediatric trauma scale (PTS).

\section{MATERIAL AND METHODS}

In this descriptive cross-sectional study, 151 consecutive cases of trauma in children younger than 17 years of age who were admitted to the emergency department of a teaching hospital in Tehran, Iran between 2009 and 2010 were enrolled. The inclusion criterion was having multisystem trauma in which two different organ systems had injury scores greater than a minor score.

The study was reviewed and authorized by the ethical committee of Shahid Beheshti University of Medical Sciences and done in accordance with good clinical practice and the Declaration of Helsinki. Understudy variables included age, sex, accident-hospital arrival time interval, weight, airway status, systolic blood pressure, consciousness status, presence and condition of any fractures or wounds, mortality, morbidity (wound infection, complications of fractures, post operation complications and etc.), Injury Severity Score (ISS) and PTS score. All the patients were followed up to hospital discharge.

ISS is an anatomically based scoring system that classifies each injury in every body region according to its relative severity on a six point ordinal scale: 1 Minor, 2 Moderate, 3 Serious, 4 Severe, 5 Critical, and 6 Maximal 
(currently untreatable). To calculate an ISS, take the highest point in each of the three most severely injured body regions, square each one and add the three squared numbers for an ISS (ISS $=\mathrm{A}^{2}+\mathrm{B}^{2}+\mathrm{C}^{2}$ where $\mathrm{A}, \mathrm{B}, \mathrm{C}$ are the scores of the three most injured ISS body regions). The ISS scores ranges from 1 to 75 . If any of the three scores is a 6 , the score is automatically set at $75[9,10]$. The values were presented and analyzed as mean \pm standard deviation. The final statistical analysis was performed using SPSS version 18.0 software (SPSS Inc, Chicago, IL, USA) using Independent-Sample $\mathrm{T}$ and Chi-Square tests. A P value of less than 0.05 was considered significant.

\section{RESULTS}

The mean age of the patients was $8.62 \pm 4.8$ years. 117 children $(78 \%)$ were male. The mean accident-hospital arrival interval time was $134 \pm 60$ minutes. The mean weight was $28.3 \pm 5.0 \mathrm{~kg}$. The mean systolic blood pressure was $96 \pm 11 \mathrm{mmHg}$. The mean PTS and ISS were $11.0 \pm 1.7$ and $64 \pm 25$, respectively.

Twelve patients $(7.9 \%)$ suffered from morbidities. 62 patients $(41.1 \%)$ had skeletal trauma, 10 of them with open fractures. The consciousness level was altered or decreased in 14 children (9.3\%). 144 children (95.4\%) had patent normal airway. 90 children (59.14\%) were observed in the emergency ward and the others were either referred or discharged. Unfortunately seven pa- tients $(4.7 \%)$ succumbed to their injuries. Only five subjects $(5.1 \%)$ had hospital stays longer than 24 hours. Pedestrian car accidents and falling down were the most common causes of injuries $(23.3 \%$ and $21.3 \%$, respectively) (Diagram 1).

The sex, cause of trauma, wound status, age, systolic blood pressure, and weight, were not related to morbidity. The mean PTS was 11.04 that this variable was 7.58 in cases with morbidity which was significantly higher than non-morbid cases. $(\mathrm{P}=0.001)$. Prolonged accident-hospital arrival time interval, closed fracture, low consciousness level, un-maintained airway, PTS and ISS were all contributing factors to morbidity $(\mathrm{P}<0.05)$ (Table 1).

\section{DISCUSSION AND CONCLUSIONS}

Pediatric trauma continues to be one of the major threats to the health and well-being of children [10,11]. The survival of children who sustain major or life-threatening trauma depends upon good pre-hospital care, appropriate triage, and resuscitation by an experienced trauma team in an emergency center, and effective emergent surgery [12]. In our study we found that the level of consciousness, airway status and type of skeletal trauma are the factors from PTS that contributed to morbidity. Moreover, PTS had significant association with morbiddity in children who sustained trauma.

In our study we chose to register the subjects from only one centre to avoid the possible variability in the

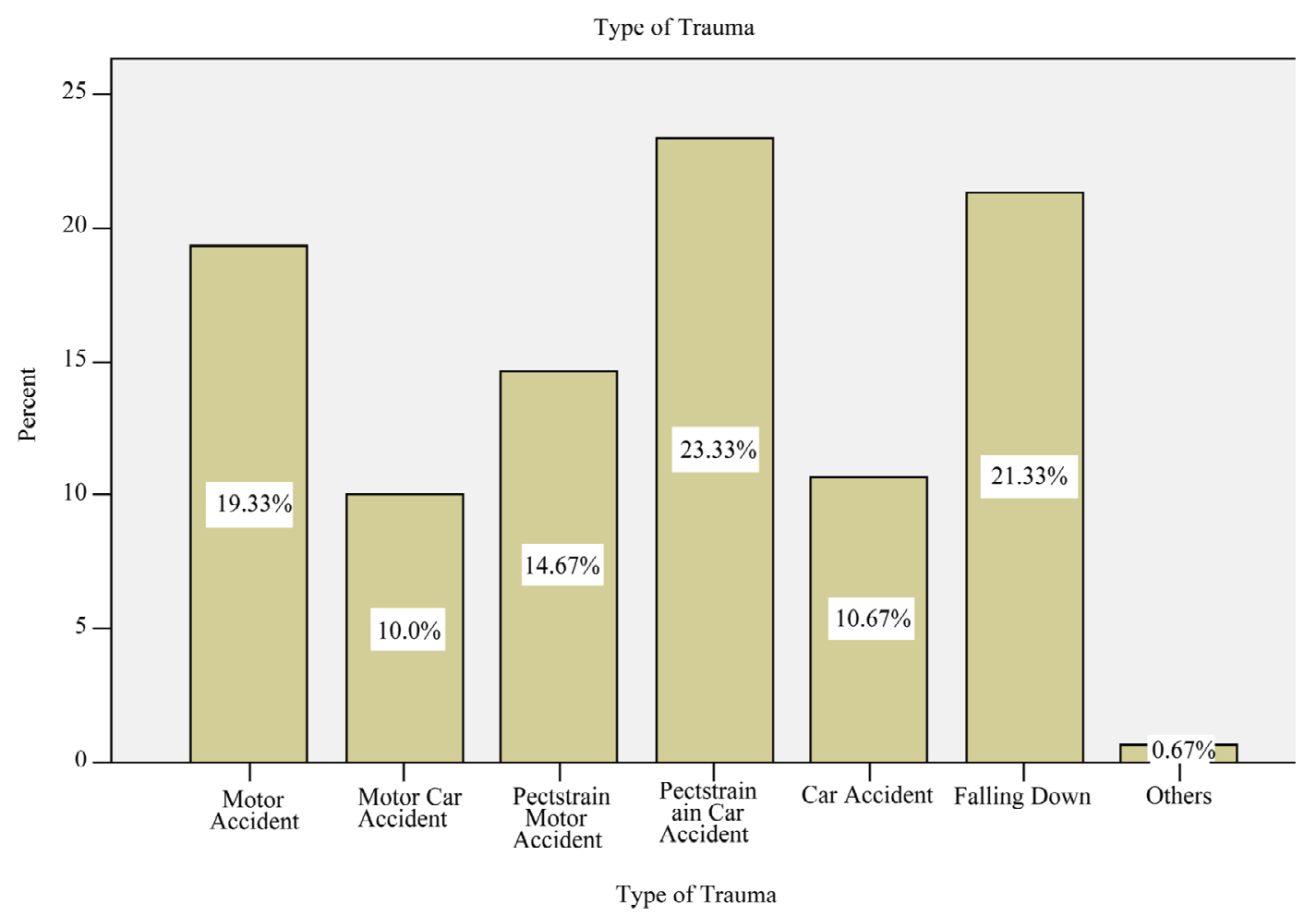

Diagram 1. Frequency type of trauma in pedestrians. 
Table 1. Contributing factors to morbidity.

\begin{tabular}{lll}
\hline Variables & Mean & P value \\
\hline Mean age (Year) & $8.62(4.84)$ & $>0.05$ \\
Trauma hospital arrival time (Min) & $134(60)$ & 0.001 \\
Mean weight (Kg) & $28.29(14.90)$ & $>0.05$ \\
Mean SBP & $96.16(11.20)$ & $>0.05$ \\
PTS $^{2}$ & $11.04(1.66)$ & 0.0001 \\
ISS $^{3}$ mean & $64.37(25.32)$ & 0.0001 \\
\hline
\end{tabular}

Abbreviations: SBP ${ }^{1}$ : Systolic Blood Pressure; PTS $^{2}$ : Pediatric Trauma Score; ISS ${ }^{3}$ : Injury Severity Score' P Value, 0.05 is significant.

triage system in different settings. While it has its own benefits, this method of data gathering makes the results of the study less generalizable to other settings. Nonetheless, our findings in this study were in line with the results of several other studies conducted in a variety of settings.

Grinkeviciute et al. in a study focused on probing the predictive value of scoring systems in severe pediatric head injury in Lithuania [13], used the PTS and GCS as the initial assessment tools. They showed that PTS $\leq 3$ and Glasgow Coma Scale (GCS) $\leq 5$ were significant risk factors of death in children with severe head injury. Potoka et al. made an effort to improve the PTS by making it age-specific [14]. To accomplish their goal, they used age-specific values for systolic blood pressure, pulse and respiratory rate for ages between 0 and 16. Interestingly, this modification improved both the sensitivity $(96.97 \%)$ and specificity $(88.83 \%)$ of this scoring system in predicting mortality.

Narci et al. studied the prognostic value of PTS in pediatric trauma patients and found it to be an independent predictor of morbidity [15].

Another setting where PTS has been used to evaluate the prognosis of severely traumatized children is the intensive care unit (ICU). Cantais et al. did a study in pediatric ICU on children with severe multiple trauma [16]. They found that, PTS $<5$, ISS $>32$ and GCS $<7$ had significant association with death in ICU setting. Matos et al. in a study in a combat support hospital in Baghdad, Iraq, with $83 \%$ penetrating trauma, showed that pediatric patients with ISS $\geq 15$, GCS $\leq 8$ and age $\leq 8$ were independently associated with mortality [17].

From this brief review of literature from a variety of settings as well as the results of our study, where blunt trauma accounts for most of the cases, we can conclude that PTS, ISS and GCS could be used as valuable prognostic tools to predict mortality and morbidity in pediatric trauma patients.

A limitation that most of the studies in this field have in common is the small sample size which negatively affects the statistical power of them and generalizability of their results. Therefore, conducting large multiple cen- ter studies can further establish pediatric injury severity scoring systems as a reliable prognostic factor for both morbidity and mortality and a useful triage tool as the same time.

\section{ACKNOWLEDGEMENTS}

This investigation was supported by a research grant from Shahid Beheshti University of Medical Sciences, Tehran, Iran.

This article was based on a postgraduate thesis by Dr. Karimian, which was successfully completed under the supervision of Dr. Derakhshanfar and Dr. Hatamabadi.

\section{REFERENCES}

[1] Behrman, R.E., et al. (2004) Nelson Textbook of Pediatrics. 17th Edition, Philadelphia, 1676-1677.

[2] Cooper, A., Barlow, B., Davidson, L., Relethford, J., O’Meara, J. and Mottley, L. (1992) Epidemiology of pediatric trauma: Importance of population-based statistics. Journal of Pediatric Surgery, 27, 149-153. doi:10.1016/0022-3468(92)90303-O

[3] Guice, K.S., Cassidy, L.D. and Oldham, K.T. (2007) Traumatic injury and children: A national assessment. Journal of Trauma and Acute Care Surgery, 63, S68-S86. doi:10.1097/TA.0b013e31815acbb6

[4] Hatamabadi, H., Vafaee, R., Haddadi, M., Abdalvand, A., Esnaashari, H. and Soori, H. (2012) Epidemiologic study of road traffic injuries by road user type characteristics and road environment in Iran: A community-based approach. Traffic Injury Prevention, 13, 61-64. doi:10.1080/15389588.2011.623201

[5] Tepas, J.J., Frykberg, E.R., Schinco, M.A., Pieper, P. and DiScala, C. (2003) Pediatric trauma is very much a surgical disease. Annals of Surgery, 237, 775-781. doi:10.1097/01.SLA.0000068118.01520.86

[6] Haley, S.M., Graham, R.J. and Dumas, H.M. (2004) Outcome rating scales for pediatric head injury. Journal of Intensive Care Medicine, 19, 205-219. doi: $10.1177 / 0885066604265798$

[7] Sise, M.J. and Sise, C.B. (2006) Measuring trauma center injury prevention activity: An assessment and reporting tool. Journal of Trauma and Acute Care Surgery, 60, 444-447. doi:10.1097/01.ta.0000196935.60165.21

[8] Buckley, J.C. and McAninch, J.W. (2006) The diagnosis, management, and outcomes of pediatric renal injuries. Urologic Clinics of North America, 33, 33-40. doi:10.1016/j.ucl.2005.11.001

[9] Furnival, R.A. and Schunk, J.E. (1999) ABCs of scoring systems for pediatric trauma. Pediatric Emergency Care, 15, 215-223. doi:10.1097/00006565-199906000-00013

[10] Kapklein, M.J. and Mahadeo, R. (1997) Pediatric trauma. Mount Sinai Journal of Medicine, 64, 302-310.

[11] Knapp, J.F. (1998) Practical issues in the care of pediatric trauma patients. Current Problems in Pediatrics, 28, 309320. doi:10.1016/S0045-9380(98)80063-7

[12] Marx, J.A., et al. (2002) Rosen's Emergency medicine: 
Concepts and clinical practice. 5th Edition, Mosby Inc., Philadelphia, 842-850.

[13] Grinkeviciute, D.E., kevalas, R., Saferis, V., Matukevicius, A., Ragaisis, V. and Tamasauskas, A. (2007) Predictive value of scoring system in sever pediatric head injury. Medicina, 43, 861-869.

[14] Potoka, D.A., Schall, L.C. and Ford, H.R. (2001) Development of a novel age-specific pediatric trauma score. Journal of Pediatric Surgery, 36, 106-112. doi:10.1053/jpsu.2001.20023

[15] Narci, A., Solak, O., Turhan-Haktanir, N., et al. (2009) The prognostic importance of trauma scoring systems in pediatric patients. Pediatric Surgery International, 25, 25-30. doi:10.1007/s00383-008-2287-5

[16] Cantais, E., Paut, O., Giorgi, R., Viard, L. and Camboulives, J. (2001) Evaluating the prognosis of multiple, severely traumatized children in the intensive care unit. Intensive Care Medicine, 27, 1511-1517. doi:10.1007/s001340101039

[17] Matos, R.I., Holcomb, J.B., Callahan, C. and Spinella, P.C. (2004) Increased mortality rates of young children with traumatic injuries at a US army combat support hospital in Baghdad, Iraq. Pediatrics 2008, 122, 959-966. doi: $10.1542 /$ peds.2008-1244 